\title{
Supersonic-to-subsonic transition of a radiation wave observed at the LMJ
}

C. Courtois, ${ }^{1, a)}$ C. Robert, ${ }^{1}$ D. Bretheau,${ }^{3}$ J. Fariaut, ${ }^{1}$ M. Ferri, ${ }^{3}$ I. Geoffray, ${ }^{2}$ G. Legay, ${ }^{2}$

F. Philippe, ${ }^{1}$ R. Rosch, ${ }^{1}$ G. Soullie,${ }^{1}$ B. Villette ${ }^{1}$

${ }^{I} C E A, D A M, D I F, F-91297$ Arpajon, France

${ }^{2} C E A, D A M, V A L D U C, F-21120$ Is sur Tille, France

${ }^{3}$ CEA, DAM, CESTA, F-33114 Le Barp, France

a)Author to whom correspondence should be addressed: cedric.courtois@cea.fr

We detail results of an experiment performed at the Laser Mégajoule (LMJ) facility aimed at studying transition from supersonic radiation front to shock front in a low density $\mathrm{CHOBr}$ foam enclosed in a plastic tube driven by thermal emission produced in a laser heated spherical gold cavity. Time resolved 2D hard x-ray radiography imaging using a Sc source (photon energy at $\sim 4.3 \mathrm{keV}$ ) is employed to measure the density perturbation front position, absorption, curvature and shocked material compression (defined as the compressed foam density normalized to its nominal value) from the supersonic to the subsonic regimes of propagation. Between these two regimes where compression goes from 1 (limited hydrodynamics) to 4 (strong shock formed), a quick increase of the foam compression is observed at the transition time ths = $6.75 \pm 0.75 \mathrm{~ns}$, corresponding to the transient transonic regime (HS means "hydrodynamically separated" and refers to the instant when the shock and the radiation front physically separate). This time is associated to a foam compression ratio of $\sim 2$ and a Mach number of the slowing down front below $\mathrm{M}<2$. Experimental results are successfully compared to 3D hydrodynamics simulations; comparisons never presented for that regime in past similar studies to our knowledge. Simulations show that the transition time ths is sensitive to the radiation closure of the tube entrance. This closure, 
which occurs in 3D, affects the amount of $\mathrm{x}$-ray energy coupled from the laser heated cavity to the $\mathrm{CHOBr}$ foam, and consequently the transient transonic regime dynamics.

\section{I.INTRODUCTION}

Radiation transfer plays an important role in Astrophysics, ${ }^{1}$ but also in the laboratory as laser energy converted into soft x-ray can be used for instance in Inertial Confinement Fusion (ICF) experiments. This is especially true in indirect drive configuration using hohlraum, ${ }^{2}$ where performances of the implosion of the capsule containing the deuterium-tritium (DT) fuel depends on the radiation field inside the cavity. The radiation field itself is governed by $\mathrm{x}$-ray energy absorption inside the cavity wall and reemission depending on wall albedo. At early time, the penetration of x-rays in the cavity wall can be assumed supersonic, ${ }^{3,4}$ that is fast enough so that heated material expansion is limited and wall density remains constant at its nominal value. Later on, cavity walls start to expand and the hydrodynamic motion affects the heat front propagation and $\mathrm{x}$-ray propagation eventually becomes subsonic. ${ }^{5,6}$

The intermediate transient transonic regime has been little studied experimentally contrary to the other two (supersonic radiation flows for instance have been extensively experimentally investigated over the last 25 years [7-14]). The transition time when $\mathrm{x}-$ ray propagation goes from supersonic to subsonic decreases with the material density $\left(\sim \rho 0^{-1.92}\right.$ for gold $)$ and it is typically in the picosecond range for solid-density gold. ${ }^{15}$ That transition time can be considerably extended, typically to the ns scale, by using low density foam or aerogel material in order to experimentally study that transition. When an incoming soft x-ray flux (photon energy $<1 \mathrm{keV}$ ) interacts with a low density foam, most of $\mathrm{x}$-ray energy is deposited in a thin layer and the material is rapidly ionized and heats which in turn reduces the opacity of the foam relative to the incident 
radiation. ${ }^{16}$ Radiation propagates then through the ionized material of constant density that has not expanded yet and is absorbed at the edge of the cold material, producing a supersonic radiation wave (Mach number, $\mathrm{M}(\mathrm{t})=\partial_{\mathrm{t} z \mathrm{~F}}(\mathrm{t}) / c_{0}(\mathrm{t})>2$ where $z \mathrm{~F}$ and $c_{0}$ correspond to the front position and the sound velocity respectively). For a qualitative analysis of the radiation front propagation, it is often assumed that the material Rosseland mean opacity, $\kappa$, the internal energy, $e$, are mainly function of its density, $\rho$, and temperature, $T$, so that they can by approximated by, $e=f T^{\beta} \rho^{-\mu}$ and $1 / \kappa=g T^{\alpha} \rho^{-\lambda} \cdot{ }^{16}$ The material pressure can be written $P=r \rho \mathrm{e}$ where, $\mathrm{f}, \beta, \mu, \mathrm{g}, \alpha, \lambda$ and $r$ are parameters that depend on the material considered. ${ }^{3}$ In a $1 \mathrm{D}$ approximation and assuming a constant drive temperature, $z \mathrm{~F} \sim\left[\sigma T_{\mathrm{S} 0}{ }^{4} \mathrm{t} /\left(\rho_{0}{ }^{2} \kappa e\right)\right]^{1 / 2}$ where $T_{\mathrm{S} 0}$ is the incident $\mathrm{x}$-ray drive and $\rho_{0}$ the initial foam density, showing the decrease of the front velocity with time, $\partial_{\mathrm{t} z \mathrm{~F}}(\mathrm{t}) \sim$ $\mathrm{t}^{-1 / 2} \cdot{ }^{16}$ Note that in our experiment, the cavity radiation temperature is not constant but increases with time so that the supersonic front position exhibits rather a linear behavior with time than the square root evolution mentioned above. The particular case where $\alpha$ $\rightarrow \infty$ (the opacity term tends towards 0 ) corresponds to a bleaching ionization wave. Its position is expected to increase linearly with time for a constant x-ray drive, ${ }^{9}$ and even accelerates when the drive increases with time, which is not what is observed in our experiment, as the supersonic front is diffusive. As the volume of heated material increases with time and x-ray drive is time limited, the supersonic radiation front then necessary slows down and material density at the front starts to build up, corresponding to the transonic regime. Note that in this regime, the radiation front is closely tied to the density perturbation and it is also characterized by a peak of plasma pressure behind the heat front. ${ }^{17}$ The time, $\mathrm{t}_{\mathrm{HS}-1 \mathrm{C} 0}$, the transonic regime is reached can be defined as the moment when the radiation front velocity, $\partial_{\mathrm{t} z \mathrm{~F}}(\mathrm{t})$, equals the sound speed $\mathrm{c}_{0}$. It can be approximated by $\mathrm{t}_{\mathrm{HS}-1 \mathrm{C} 0}=4 \Gamma g \sigma T_{\mathrm{S} 0}{ }^{4+\alpha-2 \beta} \rho 0^{-2+2 \mu-\lambda} /\left[(12+3 \alpha) \mathrm{r}(1-\mu) \mathrm{f}^{2}\right]$ when $\partial_{\mathrm{t} z \mathrm{~F}}\left(\mathrm{t}_{\mathrm{HS}-1 \mathrm{C} 0}\right)=$ 
co(thS-1 0$).{ }^{15}$ Stricter condition can be used to define transonic transition time, when $\partial_{\mathrm{t} z \mathrm{~F}}\left(\mathrm{t}_{\mathrm{HS}-2 \mathrm{C} 0}\right)=2 c_{0}\left(\mathrm{t}_{\mathrm{HS}}-2 \mathrm{C} 0\right)$, so that $4 \mathrm{t}_{\mathrm{HS}-2 \mathrm{CO}}=\mathrm{t}_{\mathrm{HS}-1 \mathrm{C} 0}$. The initial density was chosen low on purpose in our experiment, $\rho_{0}=50 \mathrm{mg} / \mathrm{cm}^{3}$ leading to values of tHS-2C $\sim 8 \mathrm{~ns}$ large enough to be explored experimentally. Note that this definition of the transition time is difficult to determine experimentally as it requires measuring the sound velocity. Another definition of ths explained below was then used instead in this study. Later on, when the subsonic regime is reached, the peak of plasma pressure behind the heat front launches a shock that propagates in the cold material and the radiation front is physically, hydrodynamically separated (HS) from it.

References $[18,19]$ describe an experiment performed on the VULCAN laser facility where the supersonic-subsonic transition is studied in a free standing lowdensity $\left(50 \mathrm{mg} / \mathrm{cm}^{3}\right)$ chlorinated foam target using a one dimensional (1D) time resolved K-shell absorption spectroscopy to infer plasma temperature and density profiles at different times. As the front Mach number is difficult to experimentally assess, the transition time, tHS, is defined when compressed material reaches twice its initial density (compression of $\rho / \rho_{0}=2$ ). Note that the subsonic regime could not be fully studied in the VULCAN experiment (maximum foam compression of 3 reached) as rarefaction waves from the side of the free standing foam reach the foam axis before the shock is fully formed, limiting the length of the foam and consequently the time over which the subsonic front can be diagnosed. Reference [20] describes an experiment performed on SG-II laser facility where shock and radiation front are studied in a free standing C8H8 foam (density of $160 \mathrm{mg} / \mathrm{cm}^{3}$ ) driven by a laser heated cylindrical gold halfraum. Shock position is studied by x-ray radiography transverse to the foam axis by looking at the decrease of the transmission of the backlighter $\mathrm{x}$-ray intensity due to the associated increase of foam density. Radiation front is studied by 
looking at its self-emission. These 1D measurements performed along the tube axis as a function of time show the dynamic of both fronts over $2.5 \mathrm{~ns}$ but no information on foam compression is presented. Yet, experimental results are compared to 1D hydrodynamic simulation showing that at early time $(t=0.25 \mathrm{~ns})$ shock compression already reached a factor of two which is the criterion introduced by Hoarty and al. to define transition time between supersonic and subsonic regime of propagation. ${ }^{18,20}$ Thus the Yan experiment mainly studies the late time transition, between transonic and subsonic regimes.

In this paper, we present results of an experiment performed at the Laser MegaJoule facility studying transition from radiation to shock wave fronts in a lowdensity $\mathrm{CHOBr}$ foam $\left(\rho_{0}=50 \mathrm{mg} / \mathrm{cm}^{3}\right)$ filling a plastic tube, using time resolved 2D hard x-ray radiography imaging. The front is driven using thermal radiation emission produced in a laser heated spherical gold cavity. From 2D radiography images, front dynamic, absorption relative to x-ray radiography source and curvature as well as shocked material compression are studied as a function of time. A quick evolution of these latter two quantities is observed in the intermediate transonic regime. The experimental setup is presented in Sec. II with target characteristics and laser beams conditions, and experimental results in Sec. III. These results are then compared in Sec. IV to results from numerical simulation obtained with the radiation hydrodynamic code TROLL run in 2D and 3D modes. A summary is given in Sec. V.

\section{EXPERIMENTAL SETUP}

Experiments were performed at the CEA/CESTA using the Laser MegaJoule. ${ }^{21}$ Each target was a millimetre scale gold spherical hohlraum coupled to a plastic tube filled with low density $\mathrm{CHOBr}$ foam (4.5\% at. $\mathrm{Br}$ doping) [see Fig. 1]. The foam composition is $46 \%$ at. of $\mathrm{C}, 46 \%$ at. of $\mathrm{H}, 3.5 \%$ at. of $\mathrm{O}$ and $4.5 \%$ at. of $\mathrm{Br}$. The length, 
diameter and density of the cylindrical foam is $2.3 \mathrm{~mm}, 2 \mathrm{R}=1 \mathrm{~mm}$ and $\rho_{0}=50 \pm 2.5$ $\mathrm{mg} / \mathrm{cm}^{3}$ respectively (average pore size diameter below $5 \mu \mathrm{m}$ ). This density was chosen to insure that the transient transonic regime lasts several ns and the density perturbation propagates over several hundreds of micrometres, compatible with the diagnostic temporal and spatial resolution. The hohlraum laser entrance hole (LEH) normal is at $90^{\circ}$ relative to the plastic tube axis.

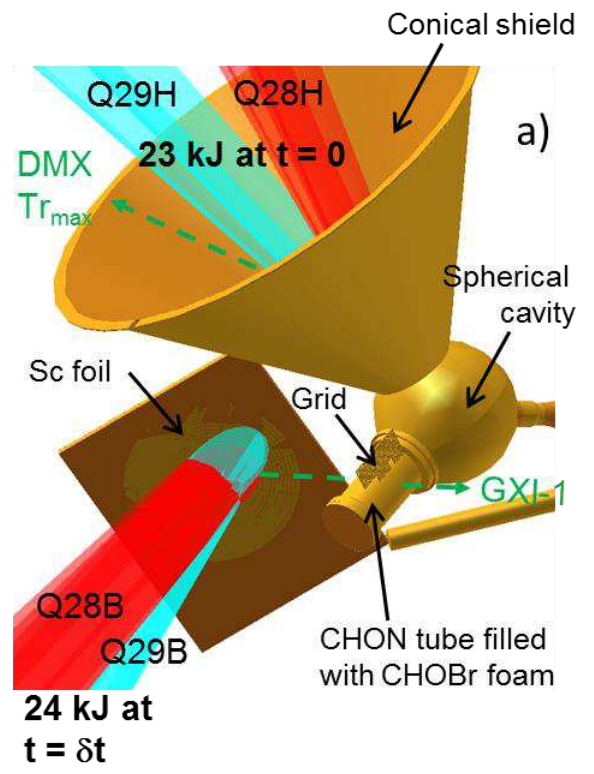

FIG. 1. Scheme showing the laser driven $\mathrm{x}$-ray source for hard $\mathrm{x}$-ray

radiography and the main target. The main target is constituted of a gold spherical cavity and a $\mathrm{CHON}$ tube containing the $\mathrm{CHOBr}$ foam. A cone is mounted around the cavity LEH to protect the tube from direct energy from the laser quadruplets Q29H and Q28H used to heat the cavity. A resolution grid mounted on the tube is used as spatial fiducial. The x-ray source is produced by focusing the laser quadruplets Q29B and Q28B on a flat scandium foil. Radiography images are recorded on the x-ray 
imager GXI-1 and the cavity radiation temperature is inferred from the broadband $\mathrm{x}$ -

ray spectrometer DMX.

A gold cone was mounted around the LEH to protect the tube from the laser energy contained in the focal spot at large radius. The tube is made of CHON and has a thickness of $90 \mu \mathrm{m}$. The CHON composition is $41.5 \%$ at. of C, $48.9 \%$ at. of $\mathrm{H}, 8.3 \%$ at. of $\mathrm{O}$ and $1.3 \%$ at. of $\mathrm{N}$. Its density is $1.2 \mathrm{~g} / \mathrm{cm}^{3}$. The position $\mathrm{z}$ is defined along the tube axis and the position $\mathrm{z}=0$ corresponds to the beginning of the foam. The thickness of the $\mathrm{CHON}$ wall was chosen large enough to maintain the soft x-ray thermal emission $(<1 \mathrm{keV})$ within the tube while allowing transmission of hard $\mathrm{x}$-ray photons from the Sc x-ray source $(\sim 4.3 \mathrm{keV})$ through it.

The spherical hohlraum is heated using 2 LMJ quadruplets (Q29H and Q28H) delivering $22 \mathrm{~kJ}$ in $3 \mathrm{~ns}$ (square pulse). One of the shot was performed at lower laser energy of $17 \mathrm{~kJ}$. These are focused with an incidence angle of $\sim 10^{\circ}$ relative to the LEH normal at the time $t=0$. The focalization is obtained with gratings that produce focal spot, around $1000 \mu \mathrm{m}$ diameter using phase plates called D-type on LMJ (circular focal spot, $1020 \mu \mathrm{m}$ diameter at $3 \%$ of the maximum intensity, approximated by a superGaussian of order 2.7) and smoothed by combining longitudinal spectral dispersion. Two other quadruplets (Q29B and Q28B, $23 \mathrm{~kJ}, 4$ ns square pulse) smoothed in the same way, are focused at $\mathrm{t}=\delta \mathrm{t}$ on a $50 \mu \mathrm{m}$-thick scandium foil, producing a strong $\mathrm{He}$ $\alpha$ x-ray emission line at an energy of $E_{\text {sc_He } \alpha} \sim 4.3 \mathrm{keV}$. These quadruplets are equipped with phase plate called E-type (circular focal spot, $1460 \mu \mathrm{m}$ diameter at $3 \%$ of the maximum intensity, approximated by a super-Gaussian of order 3.7) producing a larger and flatter laser focal spot which is suitable for hard x-ray radiography as it produces large and homogeneous x-ray source. The delay $\delta$ t was modified between shots to study 
the evolution of the density perturbation propagating inside the plastic tube. Radiography images are acquired on the 2D hard x-ray imager GXI-1 which is a gated imager equipped with four stripes that can be triggered independently at four different times. ${ }^{22}$ The target is oriented so that the radiography axis is at $90^{\circ}$ to the plastic tube axis. The 2D hard x-ray imager GXI-1, mounted inside the diagnostic insertor SID \#26, is also along the radiography axis. Images are obtained with a magnification of 4.3, with a temporal resolution of $130 \mathrm{ps}$ and a spatial resolution of $30 \mu \mathrm{m}$. Images presented in this paper are obtained with an aluminized mylar and aluminium filters. Taking into account the filter and camera spectral response, and the target transmission, the radiography images recorded by the GXI-1 are mainly due to $4.3 \mathrm{keV} \mathrm{He}-\alpha$ x-ray emission line from the Sc source as lower energy photons are absorbed.

The radiation temperature history, $\operatorname{Tr}(\mathrm{t})$, produced inside the spherical hohlraum is studied using the broadband x-ray spectrometer DMX positioned at an angle of $29^{\circ}$ relative to the LEH normal. ${ }^{23}$ In this experimental configuration, DMX measures the $\mathrm{x}$ ray thermal emission from the cavity wall but also x-ray emission from the Q29H quadruplet impact on the wall, partially seen by DMX. DMX is set up with 20 channels. The first eleven use mirrors to select the soft part of the spectrum $(<1 \mathrm{keV})$. The followings channels are dedicated to the hard part of the $\mathrm{x}$-ray spectrum $(>1 \mathrm{keV})$. The maximum radiation temperature of the cavity inferred by DMX is $179 \pm 3 \mathrm{eV}$ in the nominal experimental conditions and $165 \pm 3 \mathrm{eV}$ for the shot performed at lower laser energy.

\section{EXPERIMENTAL RESULTS}

\section{A. DENSITY PERTURBATION DYNAMICS FROM THE HARD X- RAY IMAGER}


Figure 2(a) represents an example of radiography image of the CHON tube obtained at $\mathrm{t}=7.8 \mathrm{~ns}$. The density perturbation produced inside the tube, can be seen as the local increase of density, d $\rho$, due to shock compression, increases the absorption of photons from the x-ray source. The new density reached is then written $\rho=\rho_{0}+d \rho$, where $\rho_{0}=50 \mathrm{mg} / \mathrm{cm}^{3}$ is the nominal foam density. The resolution grid used to quantify the position of the shock front is also clearly visible. Figure 2(b) shows a spatial profile obtained along the tube axis from Fig. 2(a) (black profile). The red profile is obtained from a shot performed without the main target, with GXI-1 imaging then only the x-ray source. It shows the spatial profile, the envelope of the x-ray source alone, performed in the same direction as the black profile. The red profile is normalized to the black one for shape comparison. The drop of signal on the black profile is due to a local rise of the density of the shocked and compressed $\mathrm{CHOBr}$ foam which increases the absorption of photons from the x-ray source. The position of the density perturbation, measured from the beginning of the foam $(\mathrm{z}=0)$, is defined here as the position of the signal drop. Figure 2(c) represents the measured (symbols) and the simulated (lines) positions of the density perturbation along the tube axis at different times. The black and grey symbols were obtained with a nominal and low laser energy shot respectively. Figure 2(c) also shows an example of radiography image obtained at early time $(t=2.3 \mathrm{~ns})$, during the supersonic phase of front propagation, when the density perturbation is too low to be observed experimentally by $\mathrm{x}$-ray radiography. To further constrain numerical simulations, the transmission of the density perturbation is presented in the following section. 

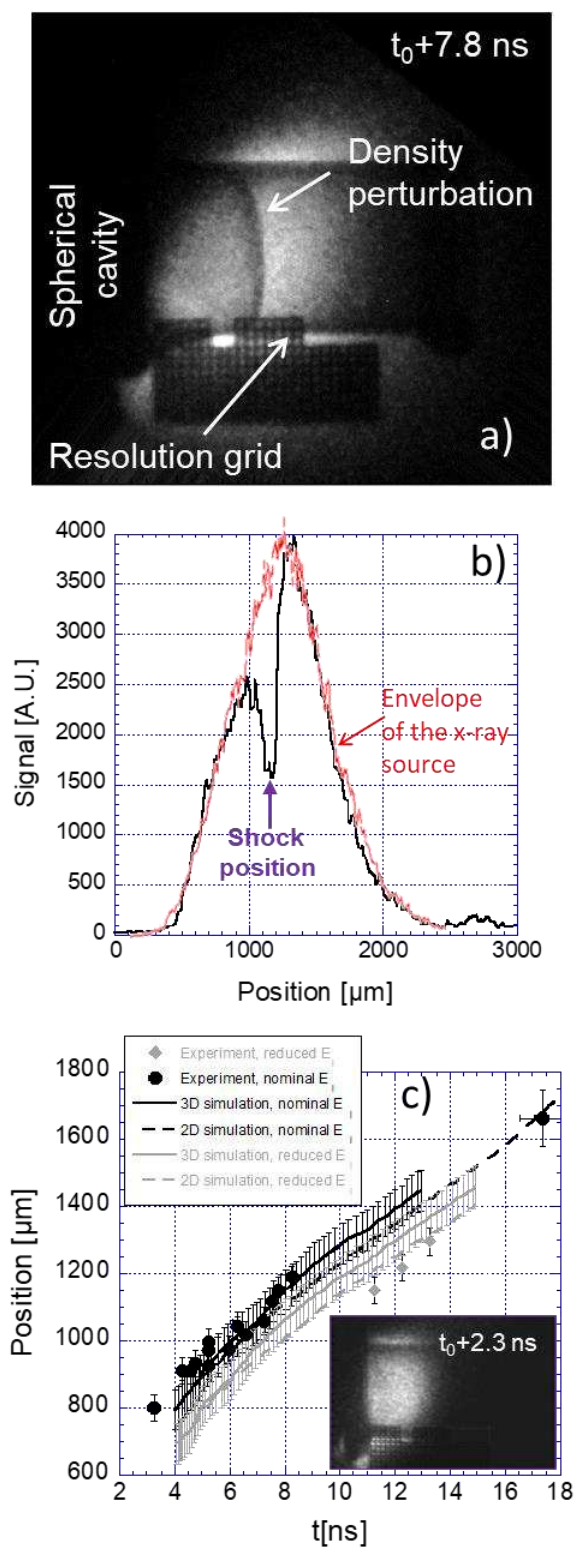

FIG. 2. (a) Radiography image of the $\mathrm{CHON}$ tube at $\mathrm{t}=7.8 \mathrm{~ns}$ showing the density perturbation due to the compressed $\mathrm{CHOBr}$ foam and the resolution grid. The 


$$
\begin{aligned}
& \text { spherical cavity is on the left of the image and the front propagates from the left to the } \\
& \text { right. (b) Spatial profiles obtained from Fig. 2(a) along the tube axis (black line) and } \\
& \text { from a similar image obtained for a shot performed without the tube (red line). (c) } \\
& \text { Position (symbols and lines correspond to experimental and simulation results } \\
& \text { respectively) of the density perturbation on the tube axis as a function of time. The } \\
& \text { inset image obtained at } \mathrm{t}=2.3 \text { ns shows that the propagation of the front is still in the } \\
& \text { supersonic regime at that time and that no density perturbation can be seen on the } \\
& \text { radiography image. }
\end{aligned}
$$

\section{B. X-RAY TRANSMISSION OF THE DENSITY PERTURBATION}

The transmission of the density perturbation can't be easily directly measured from the drop of signal observed on the radiography images [see Fig. 2(b)] as signal strength is dominated by the spatial variation of the $\mathrm{x}$-ray source. To overcome that issue, images of the x-ray source at several times were acquired without the main target. 2D map of the tube transmission is then obtained by dividing a tube radiography image as the one presented on Fig. 2(a) by the corresponding image of the x-ray source alone obtained at the same time. Figure 3(a) represents an example of such tube transmission map, here at $t=6.2 \mathrm{~ns}$. The blue profile of Fig. 3(b) is a transverse spatial profile obtained on Fig. 3(a) at a location ahead of the density perturbation, that is in a region of uncompressed foam. This profile is compared to an expected theoretical one (black profile) obtained after Abel transform of the tube knowing its nature, geometry and the characteristic of the x-ray source. These two profiles are similar, excepted at the position $\mathrm{y} \sim+500 \mu \mathrm{m}$ because of the resolution grid that affects the profile. The limited resolution of the $\mathrm{x}$-ray imager $(\sim 30 \mu \mathrm{m})$ also explains the slight discrepancy at $\mathrm{y} \sim$ $500 \mu \mathrm{m}$. The drop of transmission around $\mathrm{y}= \pm 500 \mu \mathrm{m}$ is due to the absorption of the 
tube $\mathrm{CHON}$ wall. Because of the $\mathrm{CHOBr}$ foam filling the tube, the transmission is relatively flat between $\mathrm{y}=-400 \mu \mathrm{m}$ and $\mathrm{y}=+400 \mu \mathrm{m}$. The red curve represents a longitudinal spatial profile performed along the tube axis. The transmission is relatively flat for $\mathrm{z}>700 \mu \mathrm{m}$ (noted $T_{\text {un-shocked }} \sim 25 \%$ ) since it corresponds to the region of uncompressed uniform foam. This flat section of the profile and the fact that the blue profile is close to the expected one indicates that images division performed to get the 2D transmission map is a valid approach as the $\mathrm{x}$-ray source exhibits little variations from shot to shots. This is likely explained by the fact that it is produced by 2 quadruplets, that is 8 smoothed laser beams reducing the effect of statistical fluctuations of a single beam on the overall $\mathrm{x}$-ray image. 

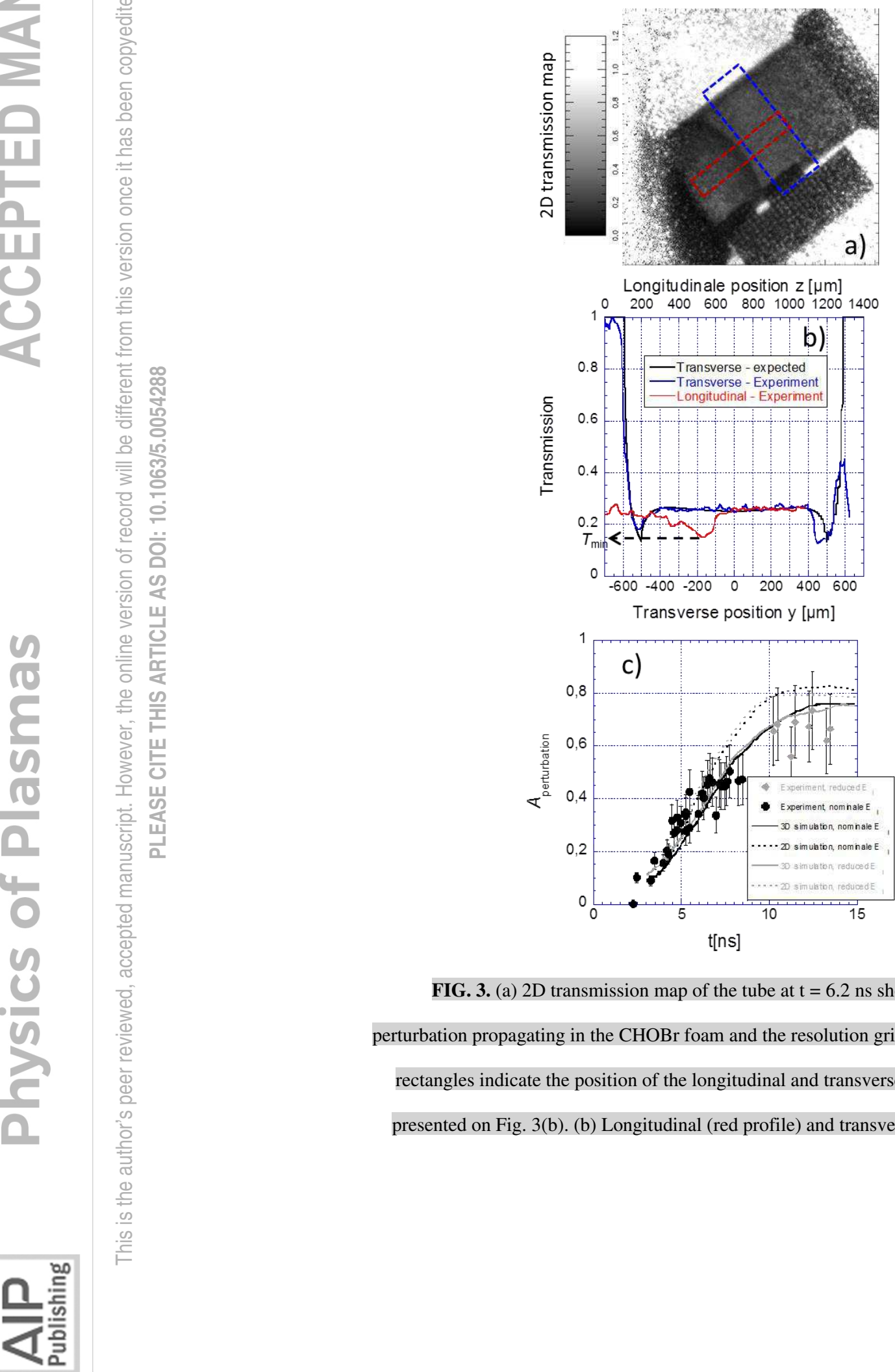

FIG. 3. (a) $2 \mathrm{D}$ transmission map of the tube at $t=6.2$ ns showing the density perturbation propagating in the $\mathrm{CHOBr}$ foam and the resolution grid. The red and blue rectangles indicate the position of the longitudinal and transverse spatial profiles presented on Fig. 3(b). (b) Longitudinal (red profile) and transverse (blue profile) 
spatial profiles obtained from Fig. 3(a). The black profile corresponds to theoretical results obtained after Abel transform of the tube. The discrepancy with experimental results (blue line) at $\mathrm{y} \sim \pm 500 \mu \mathrm{m}$ is due to the presence of the resolution grid and to the limited spatial resolution of the x-ray imager. (c) On-axis absorption due to the density perturbation as a function of time. Black and grey symbols correspond to experimental results, solid and dotted lines to 3D and 2D simulation respectively.

Black and grey colors are associated to the nominal and reduced laser energies respectively.

The density perturbation appears as a minimum transmission noted here $T_{\min }$. This minimum is due to $\mathrm{x}$-ray source photon absorption by the compressed $\mathrm{CHOBr}$ foam but also by the CHON wall. Because of the curvature of the density perturbation front, it is assumed that the $\mathrm{CHON}$ wall directly above it is unaffected so that on-axis perturbation relative absorption can be written $A_{\text {perturbation }}=\left(T_{\text {un-shocked }}-T_{\min }\right) / T_{\text {un-shocked. }}$ As it will be shown later in the simulation part, plasma wall actually suffers from x-ray preheating leading to its expansion toward the tube axis. It is assumed that the wall areal mass (the mass density spatially integrated over the path of the x-ray photons from the Sc source) remains constant. From Fig. 3(b) one gets $A_{\text {perturbation }}=48 \%$ at $\mathrm{t}=6.2$ ns. Figure 3(c) shows $A_{\text {perturbation }}$ as a function of time. Experimental and simulated results are represented by the symbols and lines respectively. At the time around $t=2.3 \mathrm{~ns}$ corresponding to the inset image presented on Fig. 2(c), the propagation of the front is still in the supersonic regime and no density perturbation can be seen on the radiography image. The point at $\mathrm{t}=2.3 \mathrm{~ns}$ is then set to $A_{\text {perturbation }}=0$ since no absorption of the photons from the $\mathrm{x}$-ray source is observed. Absorption then increases linearly with time as the density perturbation $\mathrm{d} \rho$ increases with the compression of the $\mathrm{CHOBr}$ foam, until 
it saturates beyond $\mathrm{t}=10 \mathrm{~ns}$. At this late time, a strong shock is formed in the tube and the subsonic regime is reached. The maximum foam compression is then $\rho / \rho_{0} \sim 4$ [see Sec. IV] and absorption saturates until foam decompression after target explosion (not presented here). This late time regime can be studied here, unlike past experiments that used free standing foam, thanks to the tube that prevents rarefaction waves from the edges of the foam to dislocate the target before the subsonic regime is reached.

\section{CURVATURE OF THE DENSITY PERTURBATION FRONT}

Figure 4(a) represents a zoom of the 2D transmission map of the tube at $t=6.8$ ns and a contour detection of the density perturbation front using a Canny (intensity gradient edge detection performed after noise reduction of the image) method (white curve).
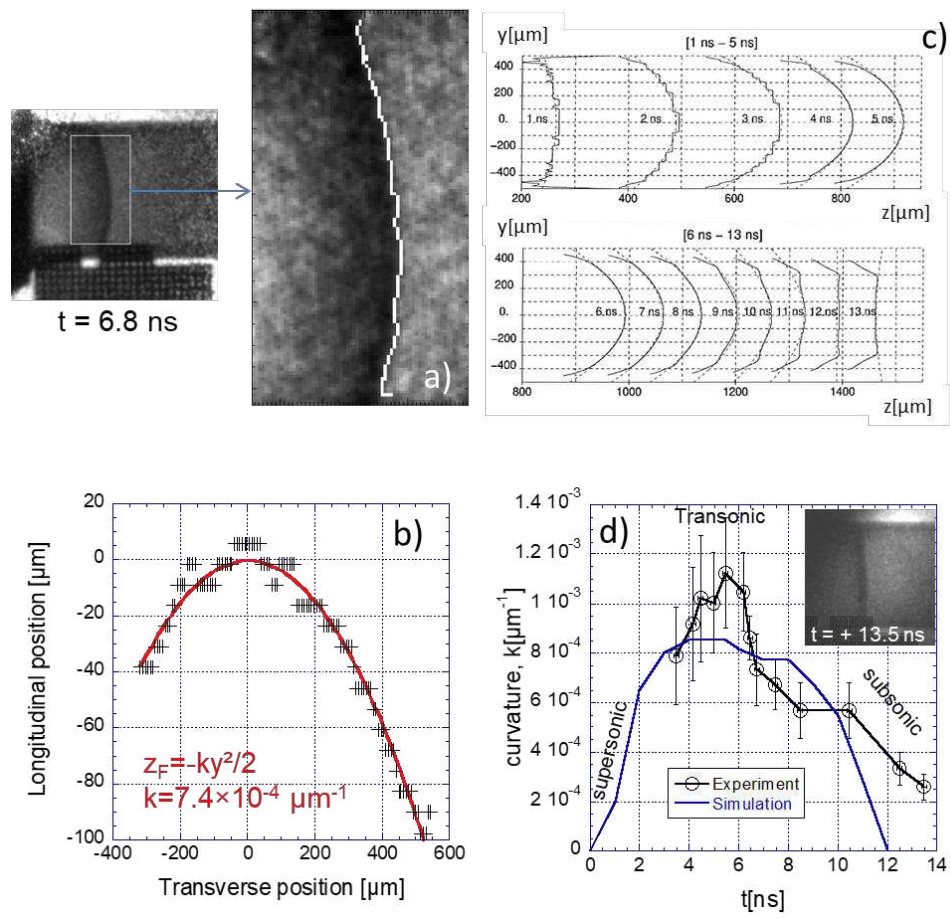
FIG. 4. (a) Contour detection of the $2 \mathrm{D}$ transmission map of the tube at $\mathrm{t}=6.8$ ns. (b) Measured longitudinal and transverse position (symbols) of the density perturbation front approximated by a parabolic profile (red solid line). (c) Simulated longitudinal and transverse position (solid line) of the density perturbation front approximated by a parabolic profile (dotted line) at different times. (d) Measured (symbols) and simulated (blue line) curvature of the density perturbation front as a function of time. Simulation results are from the radiation hydrodynamic code TROLL run here in the 2 dimensional (2D) mode. The inset image illustrates the

\section{flattening of the front at late time.}

Figure 4(b) shows the extracted longitudinal and transverse positions of the front from the zoom of Fig. 4(a) (the longitudinal coordinate is here centred at the on-axis front position). In a paper describing laser produced Marshak wave propagation in low density Ta2O5 foam $\left(\rho_{0}=40 \mathrm{mg} / \mathrm{cm}^{3}\right)$ or $\mathrm{SiO} 2$ aerogel $\left(\rho_{0}=50 \mathrm{mg} / \mathrm{cm}^{3}\right)$ contained inside Au tubes, curvature of the radiation front due to radiation energy losses at the wall tube is discussed. ${ }^{24}$ The longitudinal position of the front is written as a function of time and the transverse position y as

$$
z_{F}(y, t)=\frac{R}{\sqrt{\varepsilon}} \cosh ^{-1}\left[\frac{D \varepsilon t}{2 R^{2}}+1\right] \cos \left(\frac{\sqrt{\varepsilon}}{R} y\right)
$$

where $\mathrm{D}$ is a modified radiation diffusion constant, $\varepsilon$ a dimensionless parameter related to the wall albedo, $a$, by $\varepsilon=\rho \kappa R(1-a)$, and $\mathrm{R}$ the radius of the tube. $\rho$ is the foam density and $\kappa$ its opacity $\left(1 / \kappa=g T^{\alpha} \rho^{-\lambda}\right)$. Assuming the curvature is weak (small parameter $\varepsilon$ ), the density front can be approximated by a parabolic shape:

$$
z_{F}(y, t) \sim Z_{F}(y=0, t)-\frac{1}{2} y^{2} \frac{\varepsilon \sqrt{\mathrm{Dt}}}{R^{2}}=z_{F}(y=0, t)-\frac{1}{2} y^{2} \mathrm{k}
$$


where the curvature is described here with the parameter k. Following these results, a parabolic fit (where $\mathrm{ZF}(\mathrm{y}=0, \mathrm{t}=6.8 \mathrm{~ns})$ is set at 0 ) of the experimental results presented on Fig. 4(b) was performed (represented here by the red line). At that time, the curvature parameter is $\mathrm{k}=7.4 \times 10^{-4} \mu^{-1}$. Experimental results show that extracted front profiles can actually be easily fitted with a parabolic shape described at all time with the parameter $\mathrm{k}(\mathrm{t})$. Note that in reference [24], the description of the bending of the Marshak wave due to lossy walls applies to diffusive and supersonic radiation transport in low density medium. In the present Laser MegaJoule experiment studying transition from transonic ionization front to subsonic shock wave in low density foam, the nature of the observed front curvature can be different. Yet the parabolic description of the curvature still seems to work. From Eq. (2), a fit of the curvature parameter k with time (up to $\mathrm{t}=6 \mathrm{~ns}$ ), $\mathrm{k}=\frac{\varepsilon \sqrt{\mathrm{D}}}{R^{2}} \sqrt{\mathrm{t}}$ gives $\varepsilon \sqrt{\mathrm{D}}=112.5 \mu \mathrm{m} / \mathrm{ns}^{1 / 2}$. The front position represented on Fig. 6(b) was then fitted by the expression (1) (with y $=0$ ) which, knowing $\varepsilon \sqrt{D}$, leads to $\varepsilon \sim 0.25$ and $\mathrm{D}=2.1 \times 10^{5} \mu \mathrm{m}^{2} / \mathrm{ns}$. Using $\varepsilon=\rho \kappa R(1-a)$ and $1 / \kappa=g T^{\alpha} \rho^{-\lambda}$, one gets for $T=179 \mathrm{eV}$ (peak DMX temperature) and $\rho=50 \mathrm{mg} / \mathrm{cm}^{3}$, a wall albedo of $a=$ 0.8 which is a realistic value. Note that the radiation closure of the tube entrance also contributes to the increasing curvature of the front to a certain extent so it is actually difficult to claim exact value of the albedo. Figure 4(c) represents results from numerical simulations where simulated density fronts are also successfully approximated using a parabolic fit. Figure 4(d) shows the measured (symbols) and simulated (blue line) time evolution of the curvature parameters k. The radiography image of the front obtained at $t=13.5 \mathrm{~ns}$ shows that the front curvature is indeed less important than at $\mathrm{t}=6.8 \mathrm{~ns}$ for instance. 


\section{COMPARISON BETWEEN TROLL HYDRODYNAMICS SIMULATIONS AND EXPERIMENTAL RESULTS}

Numerical simulations of the laser produced radiation and shock fronts are performed with the Lagrangian radiation-hydrodynamics code TROLL ${ }^{25}$ used here both in $2 \mathrm{D}$ and $3 \mathrm{D}$ modes. The latter one was used to take into account more precisely the 3D aspects of the experimental configuration. 2D simulations indeed suffer from more approximations as they assume the target to be axisymmetric which is not the case in our experiment. A first simulation of the spherical cavity is then performed in $2 \mathrm{D}$ without the hole corresponding to tube position. The x-ray flux traversing the virtual position this tube is then extracted and used as an input $\mathrm{x}$-ray drive for a second $2 \mathrm{D}$ simulation of the tube that cannot then take into account properly the x-ray feeding and the radiation closure of the tube entrance. The simulations were performed in the arbitrary lagrangian-eulerain (ALE) mode. Tabulated eos and OPALV opacity ${ }^{26}$ tables are used. These were constructed from the real compositions of the materials described in section II. Simulations indicate that an artificial increase of the $\mathrm{CHOBr}$ opacity by a factor 2 shortens the front position by $80 \mu \mathrm{m}$ whereas a reduction by 2 increases the position by $100 \mu \mathrm{m}$.

\section{A. CAVITY RADIATION TEMPERATURE}

The black curve of Fig. 5 represents the radiation temperature history of the spherical cavity inferred from DMX measurement. It is compared to $2 \mathrm{D}$ and $3 \mathrm{D}$ radiation hydrodynamic simulations performed using TROLL code. 


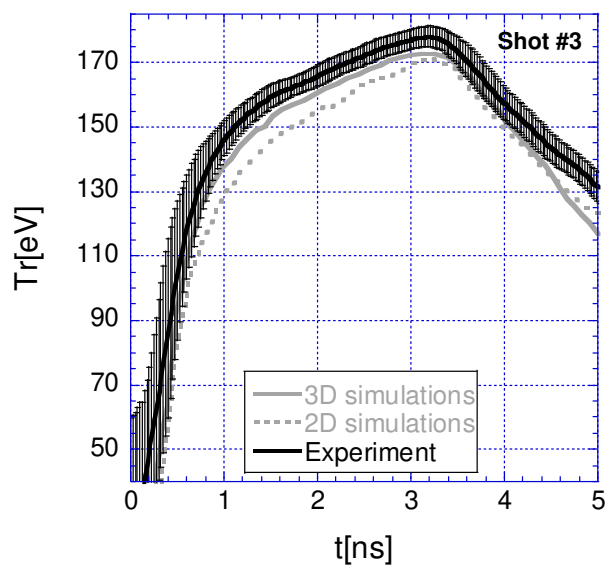

FIG. 5 Measured (black line) and simulated (grey lines) radiation temperature history of the spherical cavity for shot \#3.

A better agreement is obtained between the $3 \mathrm{D}$ simulations and the experimental results especially during the first $3 \mathrm{~ns}$ when the laser beams are on. 3D simulations can indeed take into account the real geometry of the cavity and the effective pointing of the laser beams (incidence angle of $10^{\circ}$ relative to the LEH normal). In particular, the fact that DMX can partially see the impact of Q29H on the cavity wall can explain the larger temperature obtained in $3 \mathrm{D}$ compared to the $2 \mathrm{D}$ simulation results. In $2 \mathrm{D}$ axisymmetric simulations, laser beams propagate along the LEH normal and DMX does not see the laser impact on the cavity wall. The slight discrepancy between 3D simulated and measured temperatures can be explained by laser beam pointing fluctuations. Assuming a realistic $100 \mu \mathrm{m}$ off-pointing of Q29H in a direction where beam impact on the cavity wall is more visible to DMX, brings the simulated radiation temperature history within the measured temperature error bars.

\section{B. FRONT DYNAMICS AND X-RAY ABSORPTION}


The curves of Fig. 2(c) represent the simulated position of the density front on the tube axis $(y=0)$ as a function of time. Black curves are associated to the nominal laser energy shots and gray curves to the shot performed at lower laser energy. Solid and dotted lines correspond to 3D and 2D simulations respectively. Taking into account uncertainties and fluctuations on laser energy and $\mathrm{CHOBr}$ foam density, numerical simulations indicate that the uncertainty made on the predicted position of the density front is within $\pm 60 \mu \mathrm{m}$. A relatively good agreement within the error bars is obtained between the experiment and simulations. Note that the front dynamics is faster in 3D than in 2D simulations. This is explained by a better energy coupling by $20 \%$ between the spherical cavity and the tube in $3 \mathrm{D}$, as will be discussed later. The curvature of the front shown in section III. C., is mainly governed by the x-ray energy losses through the wall (albedo $a<1$ ), at least during the supersonic regime. These loses could not be directly measured but the good agreement between experiment and simulation on the curvature suggests that the simulated wall losses are likely correct in the energy balance of the system. Note also the good agreement on the time of hydrodynamic simulation tHS (presented in the next section IV. C.) which is a quantity relatively sensitive to the temperature and density (see the section I.). The energy balance of the simulation is then likely correct so that the good agreement on the front position shown on Fig. 2(c) is effective and not due to potential compensation between wall losses and $\mathrm{x}$-ray drive at the entrance of the tube.

The curves of Fig. 3(c) represent the simulated relative absorption, $A_{\text {perturbation, of }}$ the density front on the tube axis as a function of time. Again, black curves are associated to the nominal laser energy shots and gray curves to the shot performed at lower laser energy. Solid and dotted lines correspond to 3D and 2D simulations respectively. At early time, within the first ns, absorption is low. This corresponds to 
the supersonic phase of radiation propagation characterized by a weak compression of the $\mathrm{CHOBr}$ foam. Then, absorption quickly increases and saturates when a strong shock is formed. Late time corresponds to the subsonic regime (> $10 \mathrm{~ns}$ ), when the foam compression is maximum. Intermediate time correspond to the transonic regime when the density perturbation increases with time. $2 \mathrm{D}$ and $3 \mathrm{D}$ simulations are in agreement with experimental results except during the subsonic regime where 3D simulations are closer to the measured absorption. This could be explained by a difference of treatment of the radiation closure of the tube entrance. As already mentioned, the energy coupling between the cavity and the tube is $20 \%$ higher in $3 \mathrm{D}$ simulation than in $2 \mathrm{D}$ one, consequently the transition time between the supersonic and subsonic regime is delayed in $3 \mathrm{D}$, as will be the absorption dynamics.

\section{FRONT DENSITY AND TRANSITION TIME $t_{\mathrm{HS}}$}

The symbols of Fig. 6(a) represent the on-axis $\mathrm{CHOBr}$ foam density as a function of time inferred after Abel inversion of the 2D transmission map [see Fig. 3(a)] and assuming a foam opacity $\kappa_{\mathrm{CHOBr}}=174 \mathrm{~cm}^{2} / \mathrm{g}$ for photon energy of $E_{\mathrm{sc} \_ \text {He } \alpha} \sim 4.3 \mathrm{keV}$. For $\mathrm{CHOBr}$ densities between 10 to $200 \mathrm{mg} / \mathrm{cm}^{3}$ and temperature between 10 to $80 \mathrm{eV}$, К $\mathrm{CHOBr}$ only changes by less than $10 \%$. We did not manage to infer the density perturbation experimentally from the radiography images beyond $\mathrm{t}=7 \mathrm{~ns}$ as we believe the CHON wall expansion directly above the on-axis front position can no longer be neglected. At late time, during the subsonic phase, as the shock front is relatively flat, it is no longer possible to assume that the wall directly above the front is unaffected and density estimation via Abel inversion is not possible. Solid line corresponds to results from 3D simulations. Figure 6(a) shows that at late time (subsonic regime), the maximum compression of the $\mathrm{CHOBr}$ foam is reached $\left(\rho / \rho_{0} \sim 4\right)$ corresponding to a density of $\sim 200 \mathrm{mg} / \mathrm{cm}^{3}$. At early time, during the supersonic phase of propagation, the 
density is close to the nominal density $\rho_{0}=50 \mathrm{mg} / \mathrm{cm}^{3}$ because of limited hydrodynamics. During the transonic phase, the compressed foam density increases quickly. 2D (not shown here) and 3D simulations exhibit much larger differences especially on the transition time tHs which corresponds, according to the formalism introduced by Hatchett $[18,27]$, the time when the foam compression reaches a factor $\rho / \rho_{0}=2$. Simulation results show $\mathrm{t}_{\mathrm{HS}} \sim 6.75 \pm 0.75 \mathrm{~ns}$ and $\mathrm{t}_{\mathrm{HS}} \sim 5.25 \mathrm{~ns}$ for $3 \mathrm{D}$ and $2 \mathrm{D}$ modes respectively. Similarly to the results on foam absorption presented on Fig. 3(c), the discrepancy between $2 \mathrm{D}$ and $3 \mathrm{D}$ results is consistent with a better $\mathrm{x}$-ray energy coupling between the spherical cavity and the tube in $3 \mathrm{D}$, delaying the transition time ths. 3D simulation results seem to be more compatible (within the error bars) with experimental data. The experimental transition time from supersonic to subsonic regimes is then believed to be ths $\sim 6.75 \pm 0.75 \mathrm{~ns}$. 


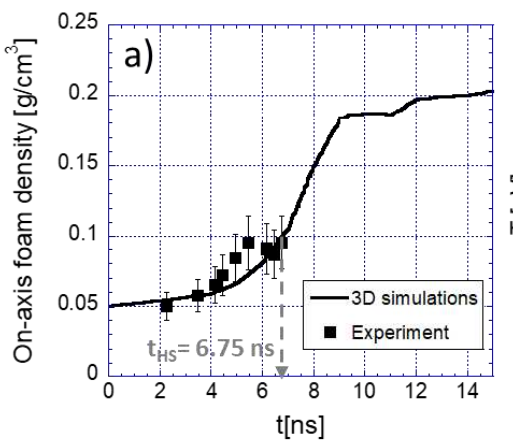

d)
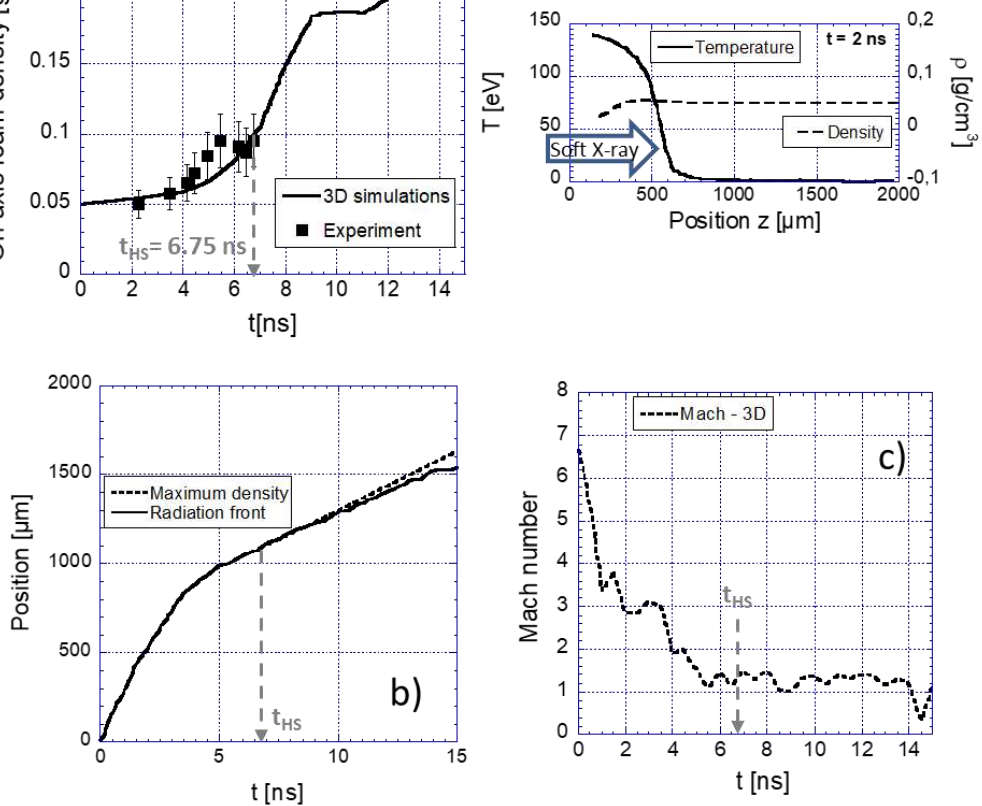

FIG. 6 a) Measured (symbols) and simulated (solid line for 3D simulations) onaxis maximum foam density as a function of time. On-axis results from 3D

hydrodynamic simulations showing $b$ ) the position of the radiation front and the maximum foam density, c) the radiation front Mach number at this front. d) On-axis $(y=0)$ density and radiation temperature profiles as a function of the longitudinal position $\mathrm{z}$ at $\mathrm{t}=2 \mathrm{~ns}$ corresponding to the supersonic regime.

Figure 6(b) represents results from 3D hydrodynamic simulation showing the position of the radiation front and the position of the "density front" as a function of time, defined as the position of the on-axis maximum density. The radiation front is defined as the inflection point of the on-axis temperature profile. At early time, during 
the supersonic regime of propagation, no density perturbation is observed and only the radiation front position is seen as shown on Fig. 6(d) where the on-axis $(\mathrm{y}=0)$ simulated density and radiation temperature profiles are represented as a function of the longitudinal position $\mathrm{z}$ at $\mathrm{t}=2 \mathrm{~ns}$. Foam density is almost at its nominal value of $\rho_{0}=$ $50 \mathrm{mg} / \mathrm{cm}^{3}$ at the front (density perturbation below 10\%) and the front temperature is around $75 \mathrm{eV}$. At the time of hydrodynamic separation ths, the two fronts (radiation and density) are closely tied. The peak pressure at the front then launches a shock in the cold material and propagates ahead of the radiation front as seen on Fig. 6(b). Because of the decrease of temperature and increase of density with time at the front, the material Rosseland mean opacity increases,${ }^{16}$ and the photon mean free path strongly decreases during the transonic regime. Simulations indicate that the photon mean free path at the radiation front is around a few of tens of microns at $t=2 n s$ then it decreases almost linearly with time and reaches few microns at ths and remains constant beyond that time. The radiation front is then basically blocked by the cold and shock-compressed $\mathrm{CHOBr}$ foam. It remains behind the "density front" but since their separation is relatively small as shown on Fig. 6(b), an instant of separation is difficult to assess. They separate from each other at a relative low speed $\sim 10 \mathrm{~km} / \mathrm{s}$, a value that could be increased using higher $\mathrm{x}$-ray drive. ${ }^{28}$ Note that electron heat conduction also contributes to energy transfer at the heat front but it also decreases with the reduction of the electron temperature. ${ }^{29}$ Figure 6(c) shows that the radiation front Mach number. It decreases because of different $\mathrm{x}$-ray leaks such as lossy wall, the reduction of the tube entrance diameter due to wall expansion. At the time of hydrodynamic separation, $\mathrm{M} \sim 1.5$ and then it slowly decreases further during the subsonic regime. In a 1D description of the problem, equations of fluids indicate that from mass and momentum conservation, a 
supersonic radiation front requires that its Mach number $M \geq 2$ otherwise a shock forms..$^{30}$

At early time ( $\mathrm{t}=2 \mathrm{~ns})$, the radiation front is then supersonic $(\mathrm{M} \sim 3)$ and also diffusive as the optical depth defined as the front position normalized to the photon mean free path is above 1. It is also interesting to evaluate the efficiency of radiation to transfer energy flux by comparing it to the enthalpy flux via the Boltzmann number, $B_{0}$ $=\left(\rho \mathrm{C}_{\mathrm{v}} \mathrm{c} T\right) /(\sigma T)$, which is below 1 in a radiative regime. ${ }^{1}$ The foam density is $\rho, \mathrm{C}_{\mathrm{v}}$ is the foam heat capacity at constant volume, the characteristic velocity for the enthalpy flux is taken as the sound velocity, $\mathrm{c}_{0}, \sigma$ is the Stefan-Boltzmann constant and $T$ is the temperature. The expression above shows that the Boltzmann number increase with the foam density and decrease with the radiation temperature. At early time, when the radiation front is at the entrance of the tube and using the cavity peak temperature (179 $\mathrm{eV}$ ), one gets $B_{0}=0.14$ showing the x-ray photons mainly carry the energy flux. Then, as the radiation front temperature decreases along the tube, the Boltzmann number increases and reaches $B_{0}=1$ for $T=T_{\mathrm{B} 0}=80 \mathrm{eV}$ (also called the critical temperature $)^{31}$ corresponding approximatively to the case presented on Fig. 6(d) showing the radiation front temperature and foam density at $\mathrm{t}=2 \mathrm{~ns}$ still in the supersonic regime (limited compression $<10 \%)$. Beyond that time, the radiation temperature continues to decrease and the density to increase [see Fig. 6(a)]. At $t=t h s$, the foam compression $\rho / \rho 0=2$ and simulation indicates that $\mathrm{T}=40 \mathrm{eV}$ at the front. The front is no longer supersonic $(\mathrm{M}<2)$ and the Boltzmann number is well above 1 meaning that the enthalpy flux dominates the energy transport.

\section{FRONT CURVATURE}

Figure 4(c) shows the edge of the density front (black solid lines) at different times from 2D simulations as it is easier to define front curvature from them. These 
edges are fitted with parabolic profiles (dotted lines) from which the curvature parameter $\mathrm{k}$ is inferred. Figure 4(d) shows the evolution of the measured (symbols) and simulated (blue line) parameter $\mathrm{k}$. Both show the same behavior with first up to $\mathrm{t} \sim 5$ ns, an increase of the curvature of the front followed by its flattening. Figure 7 shows the foam compression map for different times from the 2D TROLL simulations. The initial position of the $\mathrm{CHON}$ wall is represented by the white horizontal rectangles from $\mathrm{y} \sim 500$ to $600 \mu \mathrm{m}$. Only compression of the $\mathrm{CHOBr}$ foam is represented here and not the CHON expansion, to make the figures clearer. The increase of curvature observed on Fig. 4(d) at early time is likely due to two phenomena. During the first ns when the radiation front is supersonic, part of $\mathrm{x}$-ray energy contained in the $\mathrm{CHOBr}$ foam is lost inside the tube wall because of its low albedo, $a<1 .{ }^{24}$ This produces a slower energy transfer along the tube near the wall than on axis which tends to curve the radiation front. The second phenomenon that contributes to the bending of the front is the ablation and consequently the expansion of the wall at the entrance of the tube that ultimately reduces the x-ray energy transfer from the hohlraum to the tube and produces a point like cross section also in favor of spherical front. ${ }^{3,32}$ Since the density perturbation front is closely tied to the radiation front during the transonic regime, its curvature also increases with time. Later on, when the input drive is weakening and the density perturbation increases with the formation of a shock propagating along the tube, this shock is no longer influenced by the x-ray drive but starts to be affected by the inward expansion of the CHON tube. 

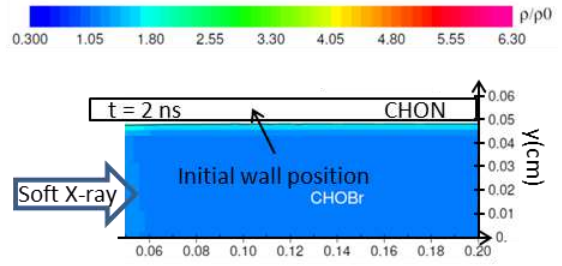

$\mathrm{z}(\mathrm{cm})$

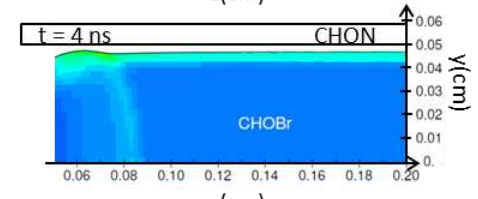

z $(\mathrm{cm})$
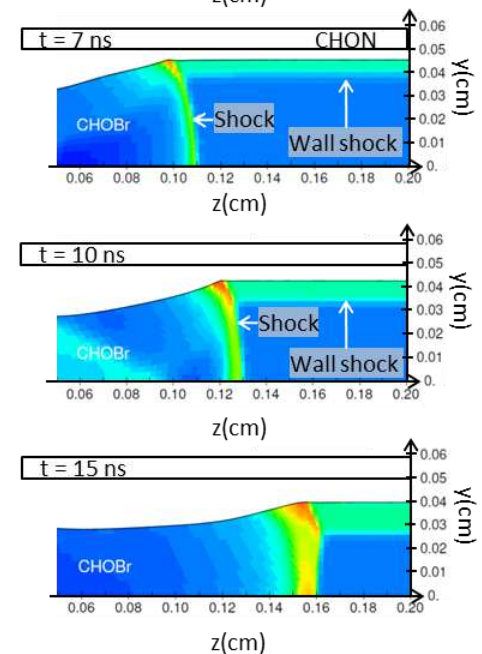

FIG. 7 Maps of the foam compression at different times from the radiation hydrodynamic code TROLL simulation run in 2D mode.

This expansion is due to early hard x-ray preheating from the gold cavity. Figure 7 clearly shows the propagation of a shock produced in the heated CHON wall and propagating inside the $\mathrm{CHOBr}$ foam, toward the tube axis. From simulations, shock wall velocity is estimated around $15 \mu \mathrm{m} / \mathrm{ns}$. The main shock due to the hydrodynamic separation in the $\mathrm{CHOBr}$ foam interacts with the wall shock and sees its shape, its curvature modified as it cannot extend to the initial wall position. ${ }^{33,34}$ The intersection 
between the main shock which distorts and the wall shock is also at the origin of a kink which is difficult to observe experimentally on the measured radiography images because of the limited contrast of the large area $\mathrm{x}$-ray radiography scheme. Yet, the change of curvature of the shock front is here clearly visible as the wall shock propagates farther towards the tube axis. Consequently, this reduces the curvature of the density front leading to smaller values of the $\mathrm{k}$ parameter that even become negative in the 2D simulations as the front goes from concave to convex shape. The simulated evolution of the $\mathrm{k}$ parameter with time exhibits a similar behavior as what is observed experimentally. The curvature first increases and then reaches a maximum before decreasing. The measured and simulated maximum value of $\mathrm{k}$ is $1.1 \times 10^{-3} \mu \mathrm{m}^{-1}$ and $0.9 \times 10^{-3} \mu \mathrm{m}^{-1}$ which are close values.

\section{CONCLUSIONS}

The objective this experiment performed on the Laser Mégajoule was to study the transition from supersonic radiation wave to shock wave fronts in low-density $\mathrm{CHOBr}$ foam in a tube driven by thermal radiation from a laser-heated spherical cavity. The growing amplitude of the foam compression during the transition from supersonic to subsonic front propagation is studied using time-resolved 2 dimensional hard $\mathrm{x}$-ray radiography transverse to the tube. Radiation front self-emission was unfortunately not measured. The dynamics, the x-ray absorption, the curvature of the density front as well as the foam compression are measured and compared successfully to 3D hydrodynamic simulations; comparisons never presented in past similar studies to our knowledge.

Results show a quick evolution of the foam compression between the supersonic regime where foam hydrodynamics can be neglected and the subsonic regime where a strong shock with foam compression of $\rho / \rho_{0}=4$ is observed (saturation of the density 
perturbation front and the absorption quantity beyond $\mathrm{t}=10 \mathrm{~ns}$ ). This transition corresponds to the transonic regime characterized by the hydrodynamic separation (HS) reached when the radiation front and the density perturbation front physically separate. At that time, the Mach number of the decelerating supersonic wave goes below $\mathrm{M}<2$ and the compression of the foam reaches $\rho / \rho_{0}=2$. The time of hydrodynamic separation is within ths $=6.75 \pm 0.75 \mathrm{~ns}$ in our experiment. Simulations show it is sensitive to the radiation closure of the tube entrance. This 3D aspect of the experiment has to be taken into account in the simulations as it governs the amount of x-ray energy coupled from the laser heated cavity to the $\mathrm{CHOBr}$ foam, and consequently the transient transonic regime dynamics.

Finally, experimental and simulation results also show an evolution of the front curvature with time affected by opposite effects: $\mathrm{x}$-ray energy losses in the tube wall (wall albedo estimated at $a \sim 0.8$ from measurements) and hydrodynamic closure of the tube entrance at early time that both increase the curvature and later on, the interaction between a shock launched from the hard x-ray preheated tube wall and the main shock propagating along the tube axis, that on the contrary tends to flatten it. Therefore, a peak of the curvature is experimentally observed at $\mathrm{t}=5 \mathrm{~ns}$.

\section{ACKNOWLEDGMENTS}

We acknowledge the support of experimental and target preparation staff of the Laser Mégajoule facility and staff of CEA/Valduc for target fabrication.

\section{DATA AVAILABILITY}

The data that support the findings of this study are available from the corresponding author upon reasonable request. 


\section{References}

${ }^{1}$ D. Mihalas and B. W. Mihalas, Foundations of Radiation Hydrodynamics (Oxford University Press, New York, 1984).

2J. Lindl, Physics of Plasmas 2, 3933 (1995).

${ }^{3}$ A.P. Cohen, G. Malamud, and S.I. Heizler1, Phys. Rev. Research 2, 023007 (2020).

${ }^{4}$ A. S. Moore, N. B. Meezan, C. A. Thomas, S. D. Bhandarkar, L. Divol, N. Izumi, A. Nikroo, T. F. Baumann, M. S. Rubery, J. Williams, N. Alfonso, O. L. Landen, W. W. Hsing, and J. D. Moody, Phys. Plasmas 27, 082706 (2020).

${ }^{5}$ R. Sigel, G. D. Tsakiris, F. Lavarenne, J. Massen, R. Fedosejevs, J. Meyer-ter-Vehn, M. Murakami, K. Eidmann, S. Witkowski, H. Nishimura, Y. Kato, H. Takabe, T. Endo, K. Kondo, H. Shiraga, S. Sakabe, T. Jitsuno, M. Takagi, C. Yamanaka, and S. Nakai, Phys. Rev. Lett. 65, 587 (1990).

${ }^{6}$ T. Shussman and S.I. Heizler, Phys. Plasmas 22, 082109 (2015).

${ }^{7}$ T. Afshar-rad, M. Desselberger, M.Dunne, J.Edwards, J. M. Foster, D. Hoarty 'M. W. Jones, S. J. Rose, P. A. Rosen, R. Taylor and O.Willi, Phys. Rev. Lett. 73, 74 (1994). ${ }^{8}$ J.Massen, G. D. Tsakiris, K.Eidmann, I. B. Foldes, Th. Lower, R. Sigel, S. Witkowski, H. Nishimura, T. Endo, H. Shiraga, M. Takagi, Y. Kato and S.Nakai, Phys. Rev. E 50, 5130 (1994).

${ }^{9}$ C. A. Back, J. D. Bauer, J. H. Hammer, B. F. Lasinski, R. E. Turner, P. W. Rambo, O. L. Landen, L. J. Suter, M. D. Rosen, and W. W. Hsing, Phys. Plasmas 7, 2126 (2000). C. A. Back, J. D. Bauer, O. L. Landen, R. E. Turner, B. F. Lasinski, J. H. Hammer, M. D. Rosen, L. J. Suter and W. H. Hsing, Phys. Rev. Lett. 84, 274 (2000).

${ }^{10}$ C. Constantin, C. A. Back, K. B. Fournier, G. Gregori, O. L. Landen, S. H. Glenzer and E. L. Dewald, Phys. Plasmas 12, 063104 (2005). 
${ }^{11}$ Y. Xu, S. Jiang, D. Lai, W. Pei, Y. Ding, T. Chang, K. Lan, S.Li, and T. Feng, Laser Part. Beams 24, 495 (2006).

${ }^{12}$ P. Keiter, M. Gunderson, J. Foster, P. Rosen, A. Comley, M. Taylor and T. Perry, Phys. Plasmas 15, 056901 (2008).

${ }^{13}$ A. S. Moore, T. M. Guymer, J. Morton, B. Williams, J. L. Kline, N. Bazin, C. Bentley, S. Allan, K. Brent, A. J. Comley, K. Flippo, J. Cowan, J. Martin Taccetti, K. MussackTamashiro, D. W. Schmidt, C. E. Hamilton, K. Obrey, N. E. Lanier, J. B. Workman, R. M. Stevenson, JQSRT 159, 19 (2015)

${ }^{14}$ T. M.Guymer, A. S.Moore, J.Morton, J. L.Kline, S.Allan, N.Bazin, J.Benstead, C.Bentley, A. J. Comley, J. Cowan, K.Flippo, W.Garbett, C.Hamilton, N. E.Lanier, K.Mussack, K.Obrey, L.Reed, D. W.Schmidt, R. M.Stevenson, J. M.Taccetti and J.Workman, Phys. Rev. Lett. 22, 043303 (2015).

15J. Garnier, G. Malinié, Y. Saillard, and C. Cherfils-Clérouin, Phys. Plasmas 13, 092703 (2006).

${ }^{16}$ J.H. Hammer and M. D. Rosen, Phys. Plasmas, 10, 1829, (2003).

${ }^{17}$ J. Pasley, P. Nilson, L. Willigale, M. G. Haines, M. Notley, M. Tolley, D. Neely, W. Nazarov, O. Willi, Phys. Plasmas, 13, 032702 (2006).

${ }^{18}$ D. Hoarty, L. Barringer, C. Vickers, O. Willi, and W. Nazarov, Phys. Rev. Lett. 82, 3070 (1999).

${ }^{19}$ O. Willi, L. Barringer, C. Vickers, and D. J. Hoarty, Astrophys. J., Suppl. 127, 527 (2000).

${ }^{20}$ Z. Ji-Yan, Y. Jia-Min, J. Shao-En, L. Yong-Sheng, Y. Guo-Hong, D. Yao-Nan, H. Yi-Xiang, and H. Xin, Chin. Phys. B 19(2), 025201 (2010).

${ }^{21}$ J.L Miquel and E. Prene, Nucl. Fusion 59, 032005 (2019). 
${ }^{22}$ R. Rosch, C. Trosseille, T. Caillaud, V. Allouche, J. L. Bourgade, M. Briat, P. Brunel, M. Burillo, A. Casner, S. Depierreux, D. Gontier, J. P. Jadaud, J. P. Le Breton, P. Llavador, B. Loupias, J. L. Miquel, G. Oudot, S. Perez, J. Raimbourg, A. Rousseau, C. Rousseaux, C. Rubbelynck, P. Stemmler, P. Troussel, J. L. Ulmer, R. Wrobel, P. Beauvais, M. Pallet, and V. Prevot, Rev. Sci. Instrum. 87, 033706 (2016).

${ }^{23}$ J. L. Bourgade, B. Villette, J. L. Bocher, J. Y. Boutin, S. Chiche, N. Dague, D. Gontier, J. P. Jadaud, B. Savale, and R. Wrobel, Rev. Sci. Instrum. 72, 1173 (2001).

${ }^{24}$ O. A. Hurricane and J. H. Hammer, Phys. Plasmas, 13, 113303 (2006).

${ }^{25}$ E. Lefebvre, S. Bernard, C. Esnault, P. Gauthier, A. Grisollet, P. Hoch, L. Jacquet,

G. Kluth, S. Laffite, S. Liberatore, I. Marmajou, P.-E. Masson-Laborde, O. Morice and J.-L. Willienoll, Nucl. Fusion 59, 032010 (2019).

${ }^{26}$ F.J.D. Serduke, E. Minguez, S.J. Davidson, C. A. Iglesias, JQSRT 65, 527 (2000).

${ }^{27}$ S. P. Hatchett, LLNL Report No. UCRL-53607, 1983.

${ }^{28}$ S. P. Hatchett, LLNL Report No. UCRL-108348, 1991.

${ }^{29}$ S. Atzeni and J. Meyer-ter-vehn, The Physics of Inertial Fusion (Clarendon, Oxford, 2004).

${ }^{30}$ J. F. Hansen, M. J. Edwards, D. H. Froula, G. Gregori, A. D. Edens and T. Ditmire, Phys. Plasmas 13, 022105 (2006).

${ }^{31}$ E. Falize, C. Michaut and S. Bouquet, The Astrophysical Journal, 730:96 (2011).

${ }^{32}$ R. R. Peterson, D. L. Peterson, R. G. Watt, G. Idzorek, T. Tierney and M. Lopez, Phys. Plasmas 13, 056901 (2006).

${ }^{33}$ F. W. Doss, H. F. Robey, R. P. Drake, and C. C. Kuranz, Phys. Plasmas 16, 112705 (2009).

${ }^{34}$ C.C. Kuranz, F.W. Doss, R.P. Drake, M.J. Grosskopf, H.F. Robey, High Energy Density Physics 6, 215-218 (2010). 


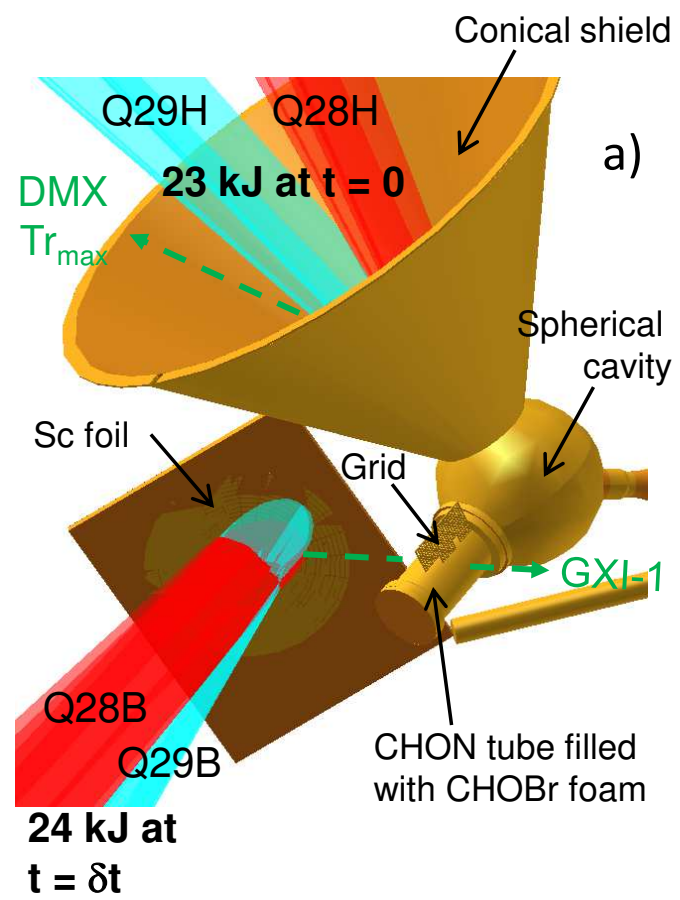

Courtois - Fig.1 

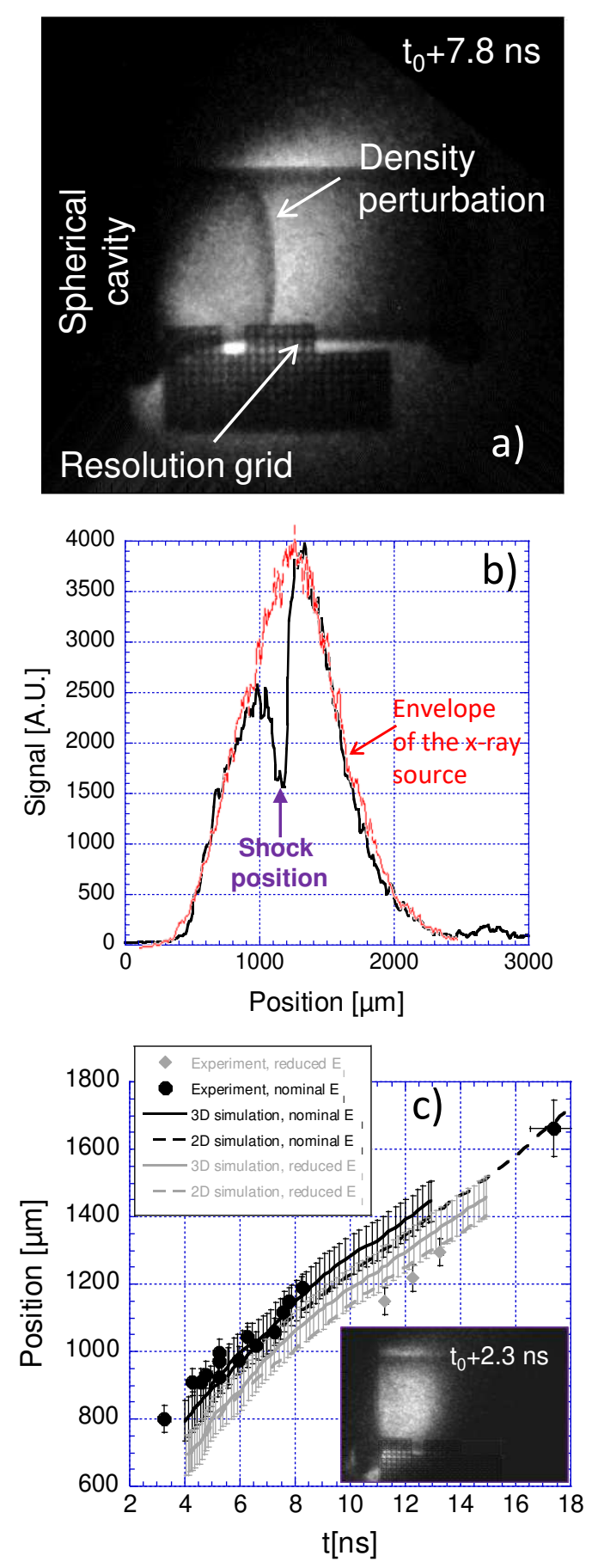

Courtois - Fig. 2 


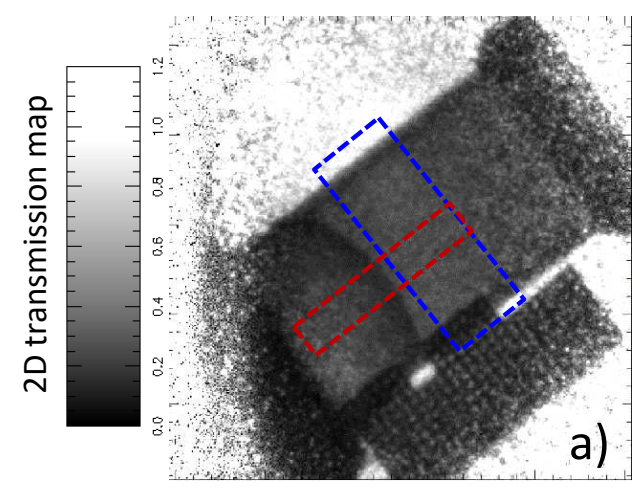

Longitudinale position $\mathrm{z}$ [ $\mu \mathrm{m}]$
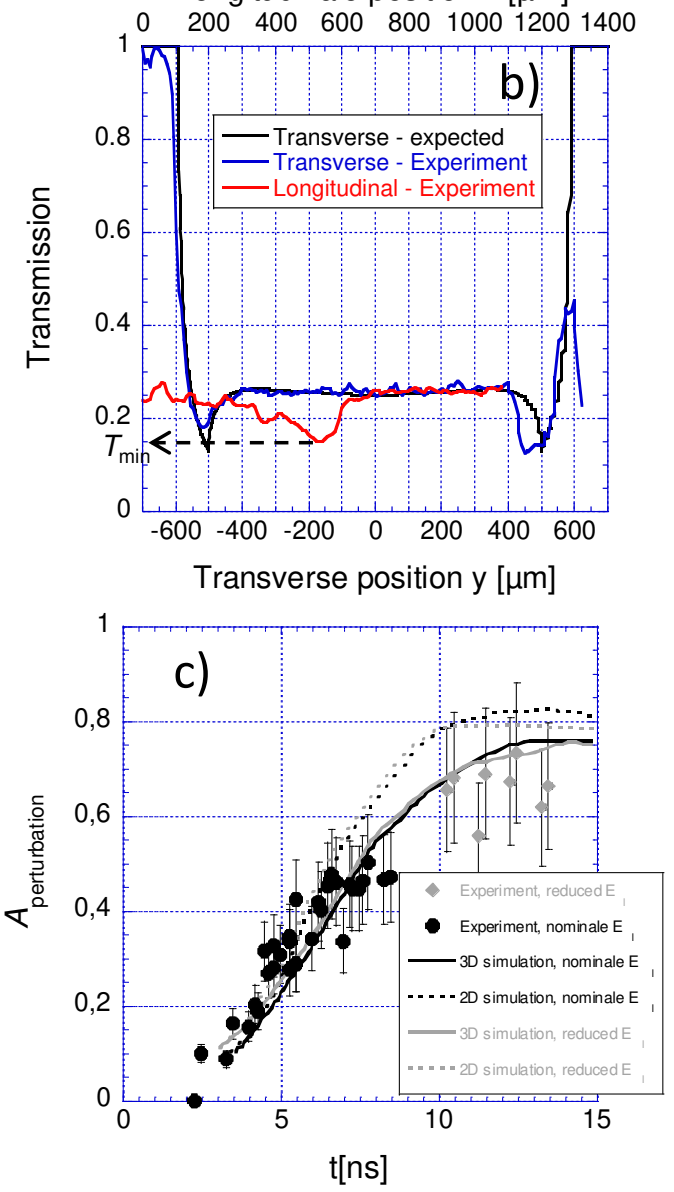

Courtois - Fig. 3 

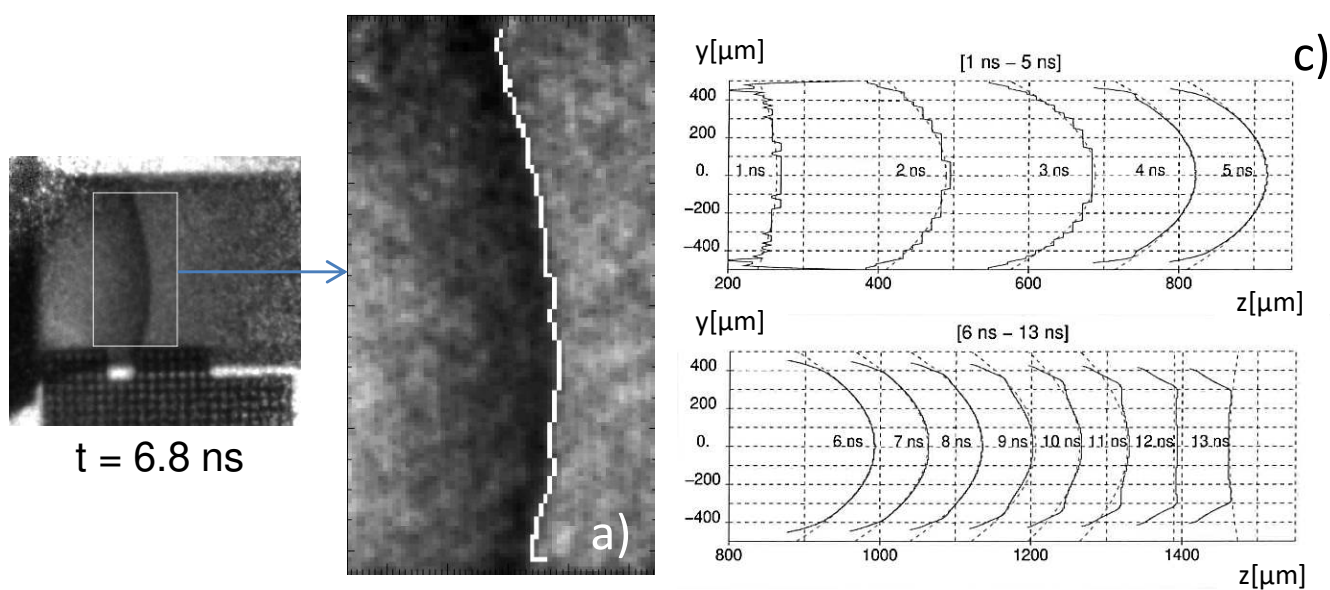

c)
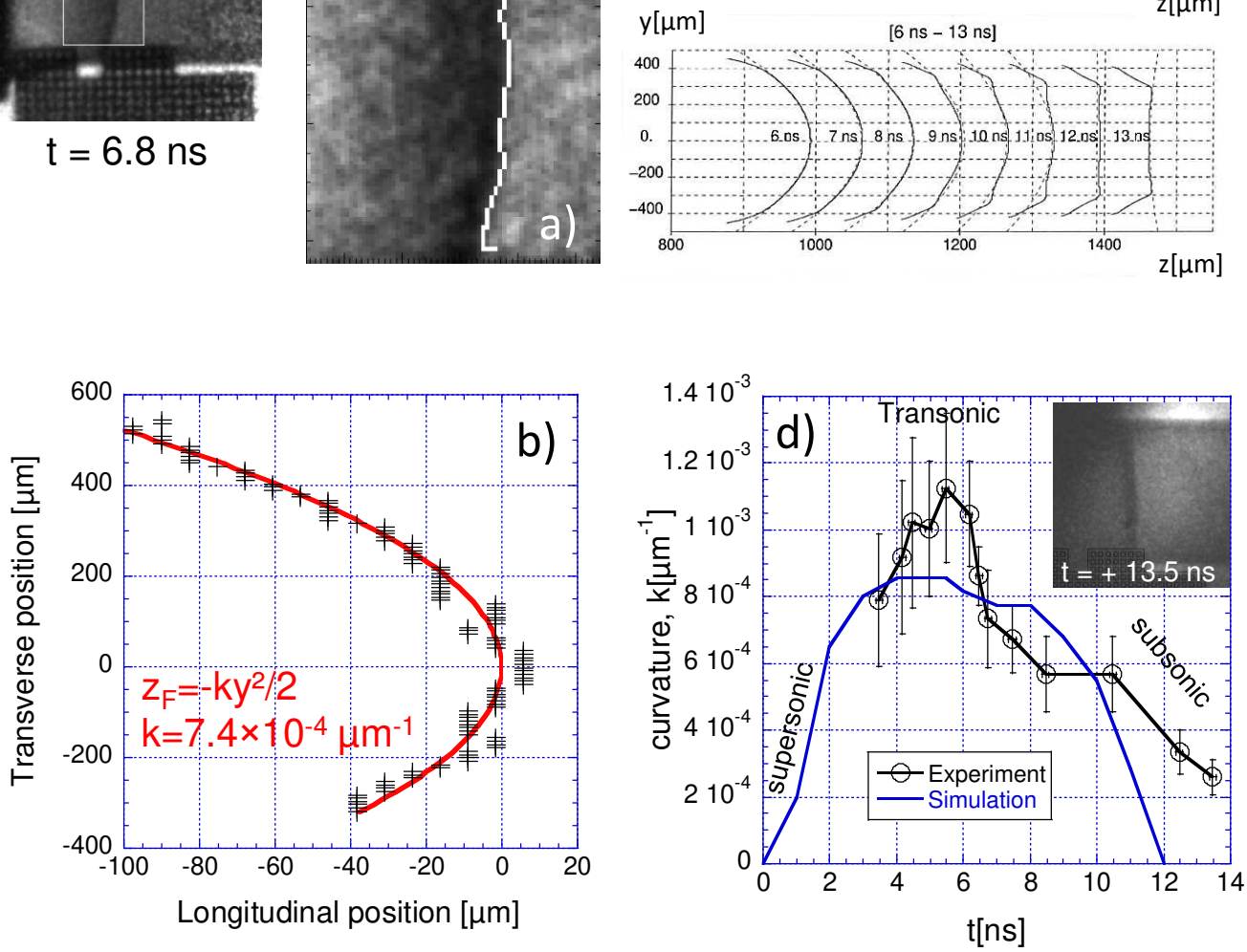

Courtois - Fig. 4 


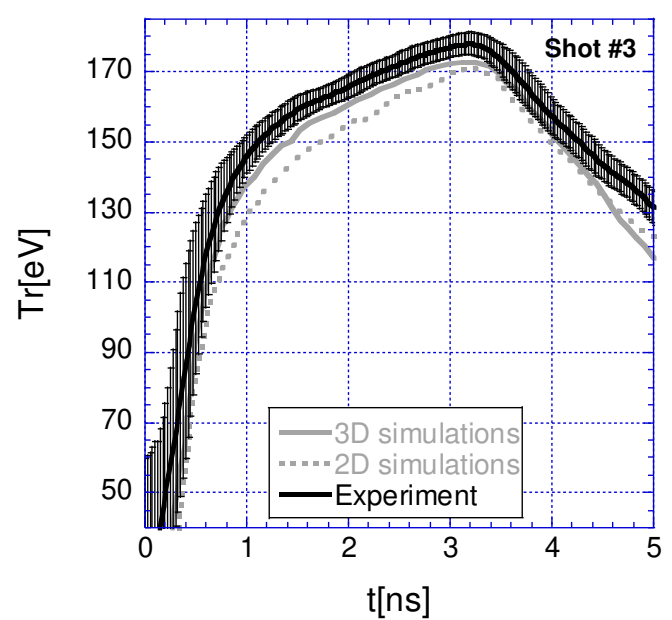

Courtois - Fig. 5 

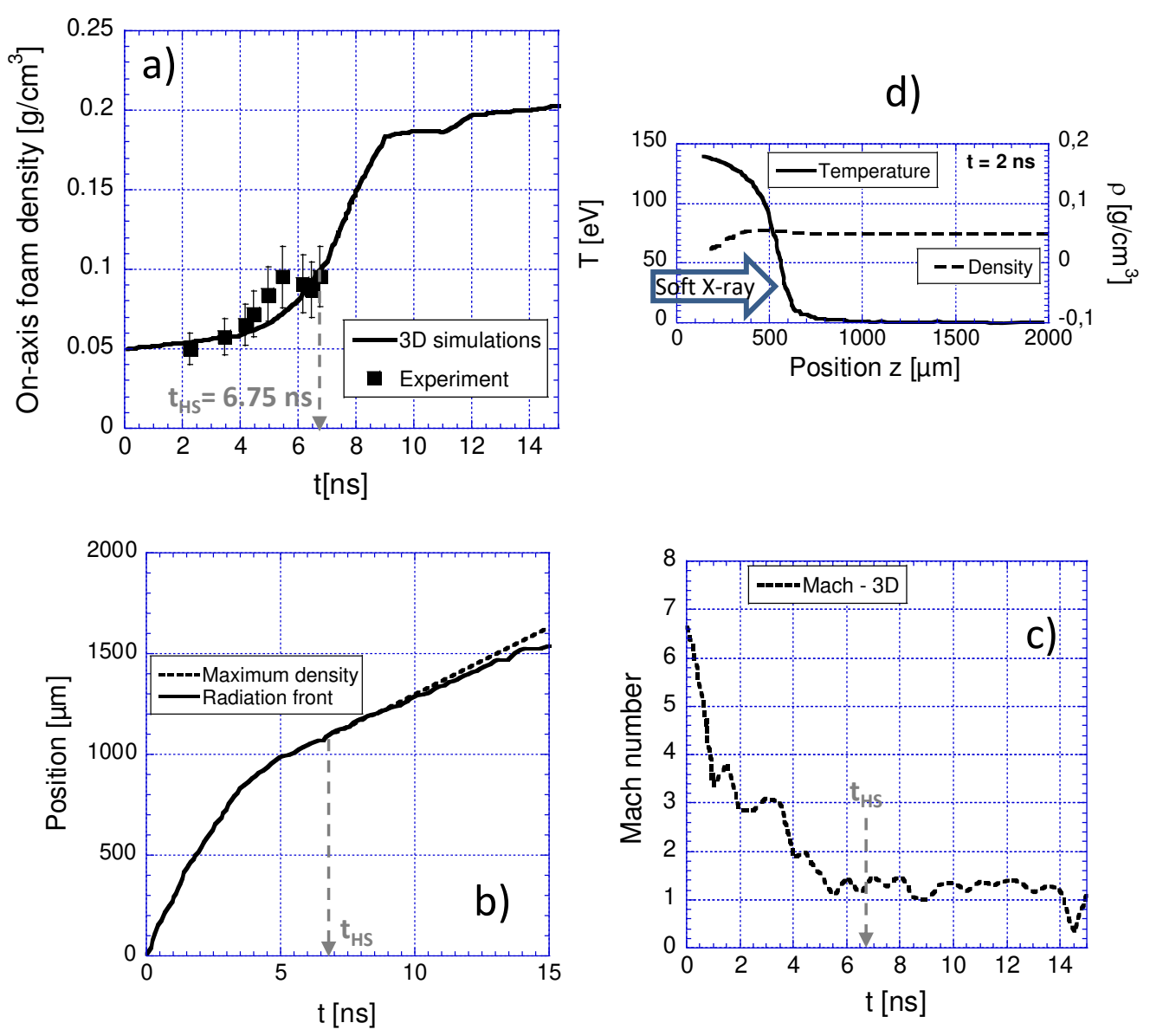

\section{Courtois - Fig. 6}




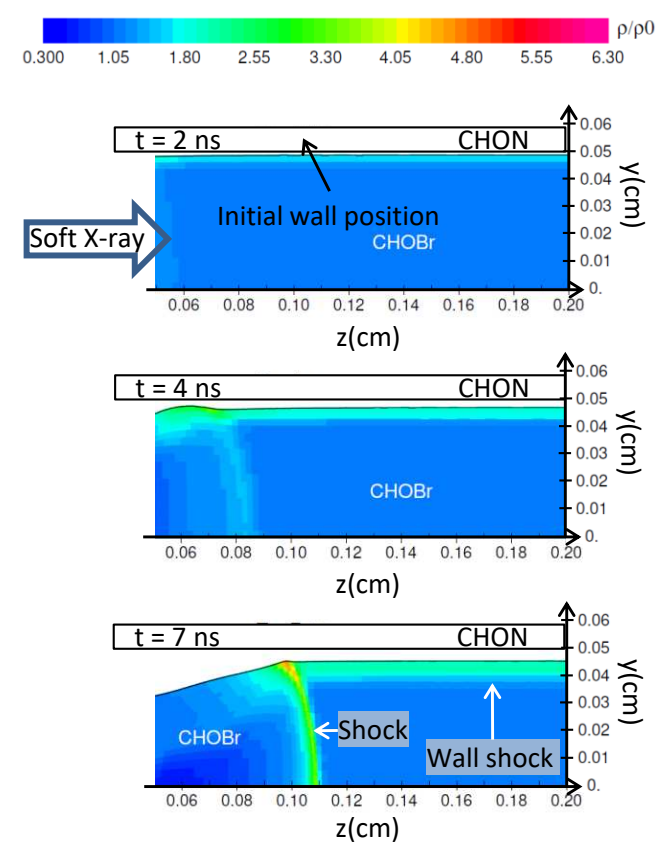

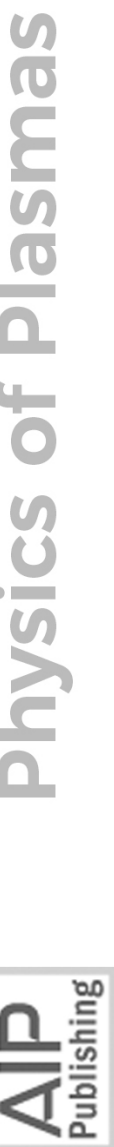

Courtois - Fig. 7 